\title{
The importance of good-quality saphenous vein segments for bypass procedures
}

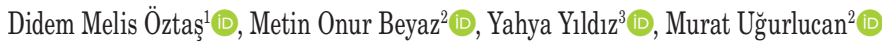

${ }^{1}$ Department of Cardiovascular Surgery, Bağcllar Training and Research Hospital, Istanbul, Turkey

${ }^{2}$ Department of Cardiovascular Surgery, Istanbul Medipol University Medical Faculty, Istanbul, Turkey

${ }^{3}$ Department of Anesthesiology and Reanimation, Istanbul Medipol University Medical Faculty, Istanbul, Turkey

We have read with great interest the manuscript entitled 'Saphenous vein graft preparation with conventional or Mayo vein stripper method: Which one is better?' written by Dr. Indelen. ${ }^{[1]}$ The study comprises some issues which need to be clarified.

The author uses second plural, i.e. 'we'; however, there is only one author listed in the manuscript. Did the sole author conduct all surgical procedures and histopathological examinations? These issues might have led to study bias. If these examinations were executed by histopathologist(s), were they blinded to the groups?

Histopathological examinations relied on basic hematoxylin and eosin staining. However, at least one additional technique, such as Mason-Trichrome staining, is mandatory to better investigate the samples. Also, we do not find any numeric values regarding studied parameters of histopathology. On the other hand, we admire the results of the author such as completely preserved vascular histological structure and no prominent leukocyte infiltration or necrosis in the interstitial tissue or lumen. Despite meticulous care, various degrees of tissue damage are inevitable. In Figure 3, the vein is pulled which may end up with tissue stretch and microvascular damage.

Furthermore, there is no information regarding the number of diseased coronary arteries, target bypass vessels, and grafted coronary arteries. It is important for the statistical analysis while comparing the number and length of the incisions and length of the saphenous veins. For instance, how could one compare the length of incisions and saphenous veins in a patient with twovessel coronary artery bypass grafting $(\mathrm{CABG})$ and in another patient who underwent four-vessel CABG? It is more complicated while comparing a $150-\mathrm{cm}$ tall patient receiving three-vessel bypass with another patient with a body length of $190 \mathrm{~cm}$ and receiving a single-vessel bypass?

Moreover, what kind of a randomization was used to classify the patients? There are no data regarding the body and leg lengths of the patients which are important to determine the lengths of the saphenous veins. The author also stated that the lengths of saphenous veins were longer in the open surgery group than the Mayo stripper method. Does the length of saphenous vein from foot to the femoral region differ according to vein graft harvesting technique? How does the harvest method influence the standard length of saphenous veins in the body? Presentation of images of the full length harvested saphenous vein segments in the manuscript would be more informative.

Correlation analysis is also mandatory to evaluate the strength of relationship between two quantitative variables. The manuscript lacks correlation analysis. Correlation of the length of saphenous vein, lengths of incisions, hazard to vein graft, or pain feeling with the harvest technique would increase the scientific power of the paper.

In the manuscript, pain was evaluated by the WongBaker Scale (Faces Pain Scale). It is widely applied in

Received: February 12, 2020 Accepted: March 12, 2020 Published online: March 25, 2020

Correspondence: Didem Melis Öztaş, MD. Bağcılar Eğitim ve Araştırma Hastanesi Kalp ve Damar Cerrahisi Kliniği, 34200 Bağclar, İstanbul, Türkiye. e-mail: didem_mls@hotmail.com 
children and rare applications in adult patients are also present. ${ }^{[2]}$ However, we failed to find the mean and standard deviation scores of pain in the manuscript. Presenting only ' $n$ ' values and percentages in the table do not give enough information about the intensity of pain with each method. Better pain and quality of life scores rather than relying on the appearance of face following painful stimuli would be more appropriate.

Additionally, there are no data regarding the infectious agents, antibiotic requirements, and duration of antibiotic use in the article.

Since the study was conducted between 2005 and 2006, to further assess the safety of both methods, at least some follow-up data regarding mortality, eventfree survival, results of angiographic studies, and re-intervention(s) would be important to evaluate each method for the vein graft patency in the long-term.

Once again, we would like to congratulate the author for the manuscript; however, the above issues should be clarified.

\section{Declaration of conflicting interests}

The authors declared no conflicts of interest with respect to the authorship and/or publication of this article.

\section{Funding}

The authors received no financial support for the research and/or authorship of this article.

\section{REFERENCES}

1. Indelen C. Saphenous vein graft preparation with conventional or Mayo vein stripper method: Which one is better?. Turk J Vasc Surg 2020;29:32-37..

2. Herr KA, Garand L. Assessment and measurement of pain in older adults. Clin Geriatr Med 2001;17:457-78.

\section{Authors' reply}

I would like to thank the authors for their valuable suggestions to my manuscript. The grafts were examined in a single-blind manner by two histopathologists. Histopathologists chose the stain type and decided to use hematoxylin and eosin. Twenty pairs of grafts were extracted from both of two groups. All grafts were unable to be included for histopathological examination due to high cost issue. All expenditures of the study were covered by myself using my own budget. I also prepare the grafts with the De Souza harvesting method and endoscopic harvesting method. Gentle surgery was required for the Mayo stripper. In Figure 3, the stripper and the graft were pushed forward together; otherwise, graft branches would be ruptured. Of note, tissue tension is related to the surgeon's experience.

The graft length is related to the patient's heart size and number of distal anastomosis. However, the number of distal sequential anastomoses is more important. A much longer graft with a short skin incision can be obtained by Mayo stripper options. In my opinion, the correlation measurement would not be beneficial for his paper, as the length of the skin incision between two groups was obvious. There was no relationship with the leg and graft length.

Two methods of harvesting were included this study. The number of total patients was divided into two groups according to gender. A code number was assigned to each other. Conventional grafts were prepared for odd numbers, and grafts were prepared for even numbers using the Mayo stripper method.

The easiest way to use the Mayo stripper is performing this method in thigh. In order to reduce the risk for infection and late wound healing, I avoid approaching the groin region by the conventional method. This may have a negative affect on the graft length in the conventional method.

For the measurement of pain, there are Visual Analog Scale, Numeric Rating Scale, and Adjective Rating Scale. The Wong-Baker Scale was preferred, as it is an easy test and extensively used in adults and children in the hospital where I work.

Infections were given as Gram-positive and Gramnegative, as only the numbers of infections were emphasized. Microorganisms identification was unable to be performed.

Angiography was performed in only nine patients which could be reached and were not included in the study.

Correspondence: Cenk İndelen, MD. Kartal Koşuyolu Yüksek İhtisas Eğitim ve Araştırma Hastanesi Kalp ve Damar Cerrahisi Kliniği, 34865 Kartal, İstanbul, Türkiye.

e-mail: cindelen@gmail.com 